\title{
Ordering for Communication-Efficient Quickest Change Detection in a Decomposable Graphical Model
}

\author{
Yicheng Chen, Rick S. Blum, Fellow, IEEE, and Brian M. Sadler, Fellow, IEEE
}

\begin{abstract}
A quickest change detection problem is considered in a sensor network with observations whose statistical dependency structure across the sensors before and after the change is described by a decomposable graphical model (DGM). Distributed computation methods for this problem are proposed that are capable of producing the optimum centralized test statistic. The DGM leads to the proper way to collect nodes into local groups equivalent to cliques in the graph, such that a clique statistic which summarizes all the clique sensor data can be computed within each clique. The clique statistics are transmitted to a decision maker to produce the optimum centralized test statistic. In order to further improve communication efficiency, an ordered transmission approach is proposed where transmissions of the clique statistics to the fusion center are ordered and then adaptively halted when sufficient information is accumulated. This procedure is always guaranteed to provide the optimal change detection performance, despite not transmitting all the statistics from all the cliques. A lower bound on the average number of transmissions saved by ordered transmissions is provided and for the case where the change seldom occurs the lower bound approaches approximately half the number of cliques provided a well behaved distance measure between the distributions of the sensor observations before and after the change is sufficiently large. We also extend the approach to the case when the graph structure is different under each hypothesis. Numerical results show significant savings using the ordered transmission approach and validate the theoretical findings.
\end{abstract}

Index Terms-Communication-efficient, CUSUM, decomposable graphical models, minimax, ordered transmissions, quickest change detection, sensor networking.

\section{INTRODUCTION}

Sensor networks are critical for many applications such as disaster response, security, smart cities, enhanced building operation for optimized energy usage, health monitoring and assisted living, and smart transportation systems [1], [2]. A fundamental problem is to detect the occurrence of a change. This can be modeled as a quickest change detection (QCD) problem, see [3]-[10] and references therein.

The work is supported by the U. S. Army Research Laboratory and the U. S. Army Research Office under grant number W911NF-17-1-0331, the National Science Foundation under Grant ECCS-1744129, and a grant from the Commonwealth of Pennsylvania, Department of Community and Economic Development, through the Pennsylvania Infrastructure Technology Alliance (PITA). Some preliminary work was presented in Y. Chen, R. Blum, and B. Sadler, "Optimal quickest change detection in sensor networks using ordered transmissions," IEEE Workshop on Signal Processing Advances in Wireless Communications, 2020.

Yicheng Chen and Rick S. Blum are with Lehigh University, Bethlehem, PA 18015 USA (email: yic917@ lehigh.edu, rblum@eecs.lehigh.edu).

Brian M. Sadler is with the Army Research Laboratory, Adelphi, MD 20878 USA (email: brian.m.sadler6.civ@mail.mil).
The classical centralized and unconstrained communication QCD problem in sensor networks is well investigated [3], [9]-[11] where each sensor monitoring the environment takes a sequence of observations. At an unknown change time, the distribution of the observations at all sensors change simultaneously. Based on the data received from the sensor nodes, a decision maker at a fusion center (FC) would like to detect the change as soon as possible subject to a false alarm constraint. Depending on knowledge of the change time distribution, Bayesian and minimax QCD formulations have been developed, and the corresponding optimal solutions are introduced in [3]. In this paper, we focus on the minimax formulation where we model the change time as a deterministic but unknown positive integer and minimize the worst case average detection delay (WADD) subject to a false alarm constraint. In many cases, each sensor in the network carries its own limited energy source, and the energy cost of communications is significant. Hence, communication efficiency is an important topic for QCD in sensor networks.

A particularly popular approach called censoring has been shown to be an effective method to improve communication efficiency where sensors transmit only highly informative data [12]. In [12], upper and lower thresholds are set and sensors transmit only very large or small likelihood ratios because these values provide significant information about which hypothesis is most likely to be true. Censoring-based QCD is proposed in [5] [13] where it is shown that censoring yields transmission savings but always increases detection delay (the accepted performance measure for QCD).

In this paper we introduce an ordered transmission QCD method that will lower communications without increasing the detection delay. The ordered transmission approach (also called ordering) was first introduced for a distributed testing problem between two fixed hypotheses and employing an FC [14]. Using ordering the sensors with the most informative observations transmit first. Transmissions can be halted when sufficient information is accumulated for the FC to decide which hypothesis is true. In [14], it was shown that this ordered transmission approach can reduce the number of transmissions without losing any detection performance for cases with statistically independent observations. The detection of a mean shift or covariance matrix change in statistically dependent Gaussian observations following a decomposable Gaussian graphical model (GGM) is considered in [15] and [16], respectively. In this paper, we provide the first communication efficient QCD algorithm for rapidly detecting a change with statistically 
dependent observations across the sensors. The focus is on the case where the observations follow a decomposable graphical model (DGM) to characterize the dependence among sensor observations, completely subsuming the case with independent observations. This work is a highly nontrivial extension of the initial work in [17], which was limited to independent observations and one-hop communications to an FC.

\section{A. Our Contributions}

This work describes a new algorithm that provides a communication efficient distributed processing method for a sensor networking change detection problem where the observations follow a statistically dependent distribution before and after the change. The focus is on the case where both distributions are characterized by a decomposable graphical model (DGM) with common graph structure. In Section IV-D, extensions are described for the case where the graph structure of the distributions before and after the change are different. The distributed computation describes the proper way to collect nodes into local groups, corresponding to the cliques 1 in the graph, such that each clique can produce a compressed clique statistic that summarizes all the clique sensor data. The clique test statistics can be transmitted to a common location and summed to produce the standard optimum centralized change detection test statistic. Distributed computation methods have not been previously described for these change detection problems as they are complicated by the statistically dependent data. With proper clique formation, we then employ ordering to reduce the number of clique test statistics that need to be transmitted without any performance loss. We derive a lower bound on the number of transmissions saved, and show that up to one half savings is possible for some cases of interest.

\section{B. Problem Formulation}

We consider a sensor network with $M$ sensors and a FC. Sensor $m$ for $m=1,2, . ., M$ observes the sequence $\left\{X_{n, m}\right\}_{n \geq 1}$ with $n$ denoting the time slot index. The objective is to detect a change as quickly as possible after the change occurs which implies the goal is to minimize detection delay if the change occurs. As long as no change is declared, the sensors will continue observing data. Throughout this paper we make the following assumption.

Assumption 1: The distributions of the observations at all sensors change simultaneously at the change time $\tau$. In particular, at the unknown time slot $\tau$, the distribution of $X_{n,[1, M]} \triangleq\left(X_{n, 1}, X_{n, 2}, \ldots, X_{n, M}\right)$ changes from $f_{0}$ to $f_{1}$ where $f_{0}$ and $f_{1}$ are the known probability density functions (pdfs) before and after the change time, respectively. The random variable $X_{n, m}$ is independent across the time slot index $n$ but will generally assumed to be dependent across the sensor index $m$.

Without a prior on the distribution of the change time, we model the change time $\tau$ as a deterministic but unknown integer and we employ the constraint

$$
\mathbb{E}_{\infty}\left(n^{\prime}\right) \geq \gamma
$$

\footnotetext{
${ }^{1} \mathrm{~A}$ clique is defined in this paper as a set of vertices that induce the largest fully connected subgraph.
}

where $n^{\prime}$ denotes the time slot when the decision maker declares a change has occurred, $\mathbb{E}_{\infty}\left(n^{\prime}\right)$ is the average delay when the change does not occur, and $\gamma$ is a pre-specified constant. If the change occurs, then one formulation to evaluate the detection delay is to employ the WADD defined in [6] as

$$
\operatorname{WADD}\left(n^{\prime}\right)=\sup _{\tau \geq 1} \operatorname{ess} \sup \mathbb{E}_{\tau}\left[\left(n^{\prime}-\tau\right)^{+} \mid \mathcal{I}_{\tau-1}\right]
$$

where ess $\sup X$ denotes essential supremum of $X, \mathbb{E}_{\tau}$ is the expectation when the change occurs at time $\tau,(x)^{+} \triangleq$ $\max \{x, 0\}, \mathcal{I}_{\tau-1} \triangleq\left(X_{[1, \tau-1], 1}, \ldots, X_{[1, \tau-1], M}\right)$ denotes past global information at time slot $\tau$, and $X_{[1, \tau-1], m} \triangleq$ $\left(X_{1, m}, \ldots, X_{\tau-1, m}\right)$ denotes past local information at sensor $m$. Thus, the QCD problem in a minimax setting can be formulated as a constrained optimization problem

$$
\begin{aligned}
& \min _{n} \operatorname{WADD}\left(n^{\prime}\right) \\
& \text { s.t. } \mathbb{E}_{\infty}\left(n^{\prime}\right) \geq \gamma .
\end{aligned}
$$

\section{Paper Organization}

The paper is organized as follows. In Section II a brief discussion on mathematical formulations of decomposable graph models is described. In Section III, we describe distributed computation of the optimum test statistic. Communicationefficient QCD using ordered transmissions is described in Section IV] and a lower bound on the average number of transmissions saved via ordering is provided. Section $\mathrm{V}$ presents some numerical results to demonstrate the communication efficiency of the proposed algorithm. Finally, we conclude the paper in Section VI

\section{Decomposable Graphical Models}

DGMs have received extensive study in machine learning [18], [19], sensor networks [20] and electric power systems [21]. In this section, we briefly describe the basic theory of DGMs. Consider an undirected graph $\mathcal{G}=(\mathcal{V}, \mathcal{E})$ with $M$ vertices, where $\mathcal{V}=\{1,2, \ldots, M\}$ is the set of vertices and $\mathcal{E}=\left\{\left(i_{1}, j_{1}\right),\left(i_{2}, j_{2}\right), \ldots,\left(i_{|\mathcal{E}|}, j_{|\mathcal{E}|}\right)\right\}$ denotes the set of undirected edges of the graph. The graphical model for a random vector $X_{i,[1, M]}$ at each time slot $i$ with graph $\mathcal{G}$ describes the statistical dependency model such that for each time slot $i, X_{i,[1, M]}$ follows a known distribution that obeys the pairwise Markov property with respect to $\mathcal{G}$. The distributed observation vector $X_{i,[1, M]}$ satisfies the pairwise Markov property with respect to $\mathcal{G}$ if, for any pair $\left(m, m^{\prime}\right)$ of non-adjacent vertices, i.e., $\left(m, m^{\prime}\right) \notin \mathcal{E}$, the corresponding pair of elements of $X_{i,[1, M]}, X_{i, m}$ and $X_{i, m^{\prime}}$, are conditionally independent when conditioned on the remaining elements. This can be expressed as

$$
\begin{aligned}
& f\left(X_{i, m}, X_{i, m^{\prime}} \mid X_{i, \mathcal{V} \backslash\left\{m, m^{\prime}\right\}}\right) \\
& =f\left(X_{i, m} \mid X_{i, \mathcal{V} \backslash\left\{m, m^{\prime}\right\}}\right) f\left(X_{i, m^{\prime}} \mid X_{i, \mathcal{V} \backslash\left\{m, m^{\prime}\right\}}\right)
\end{aligned}
$$

where we have used $f(\cdot)$ to denote the corresponding pdfs.

An undirected graph is decomposable if the graph has the property that every cycle of length larger than 3 possesses a chord [22]. Throughout the paper, we concentrate 
on decomposable undirected graphical models. Let $K$ denote the number of cliques in the decomposable undirected graph $\mathcal{G}$. The sequence of cliques of the graph $\mathcal{G}$ is denoted by $\left\{\mathcal{C}_{k}\right\}_{k=1}^{K}$. We denote the corresponding histories $\left\{\mathcal{H}_{k}\right\}_{k=1}^{K}$ and separators $\left\{\mathcal{S}_{k}\right\}_{k=2}^{K}$ as

$$
\mathcal{H}_{k}=\mathcal{C}_{1} \cup \mathcal{C}_{2} \cup \cdots \cup \mathcal{C}_{k}, \forall k=1,2, \ldots, K,
$$

and

$$
\mathcal{S}_{k}=\mathcal{H}_{k-1} \cap \mathcal{C}_{k}, \forall k=2, \ldots, K .
$$

Note that from (5), the $k$-th history $\mathcal{H}_{k}$ contains all nodes in the first $k$ cliques. The $k$-th separator $\mathcal{S}_{k}$ in (6) is the set of the common nodes between $\mathcal{H}_{k-1}$ and $\mathcal{C}_{k}$. A set is complete if it induces a fully connected subgraph [22]. For any decomposable undirected graph $\mathcal{G}$, the sequence of cliques $\left\{\mathcal{C}_{k}\right\}_{k=1}^{K}$ of $\mathcal{G}$ is said to be perfect if the following conditions are satisfied [22]

1) the sets $\mathcal{S}_{k}$ are complete for all $k=2,3, \ldots, K$;

2) for all $k>1$, there is a $j<k$ such that $\mathcal{S}_{k} \subseteq \mathcal{C}_{j}$.

The condition 2 is also called the running intersection property. Note that $\mathcal{S}_{k}$ separates $\mathcal{H}_{k-1} \backslash \mathcal{S}_{k}$ and $\mathcal{C}_{k} \backslash \mathcal{S}_{k}$ based on (5) and (6) such that all paths from the nodes in $\mathcal{H}_{k-1} \backslash \mathcal{S}_{k}$ to the nodes in $\mathcal{C}_{k} \backslash \mathcal{S}_{k}$ intersect $\mathcal{S}_{k}$.

A mapping $q:\{2,3, \ldots, K\} \rightarrow\{1,2, \ldots, K\}$ is defined to specify an association between each separator set and one unique clique such that [15]

$$
q(k) \triangleq \min \left\{j \mid \mathcal{S}_{k} \subseteq \mathcal{C}_{j}\right\}, \forall k=2,3, \ldots, K .
$$

Note that $q(k)$ describes the minimum index of a clique that contains the $k$-th separator $\mathcal{S}_{k}$. Thus, the $k$-th separator $\mathcal{S}_{k}$ is associated with the $q(k)$-th clique $\mathcal{C}_{q(k)}$ according to

$$
\mathcal{S}_{k} \subseteq \mathcal{C}_{q(k)}
$$

The $k$-th separator $\mathcal{S}_{k}$ is not only contained in the $q(k)$-th clique $\mathcal{C}_{q(k)}$, but it is also contained in the $k$-th clique $\mathcal{C}_{k}$ based on (6), that is,

$$
\mathcal{S}_{k} \subseteq \mathcal{C}_{k}
$$

It follows that for any $k>1, q(k)$ must exist and

$$
q(k)<k
$$

for any decomposable undirected graph $\mathcal{G}$. Let $\mathcal{Q}_{j}$ denote the set of indices of the separators which are associated with the $j$-th clique via the mapping $q$ in (7), that is,

$$
\mathcal{Q}_{j} \triangleq\{k \mid q(k)=j\} \text {. }
$$

Note that $\mathcal{Q}_{j}$ enumerates the separators contained in the $j$-th clique except the $j$-th separator. Thus, $\mathcal{Q}_{j} \cup\{j\}$ contains all the indices of the separators which are contained in the $j$-th clique. From 10 , we know that the minimal element in $\mathcal{Q}_{j}$ satisfies

$$
\min \mathcal{Q}_{j}>j,
$$

which implies that

$$
\mathcal{Q}_{j} \subseteq\{j+1, j+2, \ldots, K\}, \forall j=1,2, \ldots, K-1,
$$

and

$$
\mathcal{Q}_{K}=\emptyset \text {. }
$$

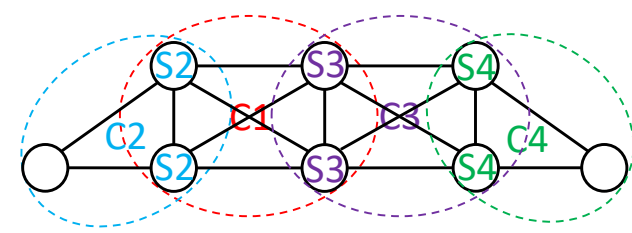

Fig. 1. The decomposable graphical model with 4 cliques and numbered separators.

1) Illustration of (5)-(14) using Fig. 17. Consider the example in Fig. 1 By employing (7), we observe that $q(2)=$ $q(3)=1$ and $q(4)=3$ which implies $\mathcal{S}_{2} \subseteq \mathcal{C}_{1}, \mathcal{S}_{3} \subseteq \mathcal{C}_{1}$ and $\mathcal{S}_{4} \subseteq \mathcal{C}_{3}$. By employing (9), we also obtain $\mathcal{S}_{2} \subseteq \mathcal{C}_{2}, \mathcal{S}_{3} \subseteq \mathcal{C}_{3}$ and $\mathcal{S}_{4} \subseteq \mathcal{C}_{4}$. As per 110$), q(2)<2, q(3)<3$ and $q(4)<4$. By employing 111 , we obtain $\mathcal{Q}_{1}=\{2,3\}$ and $\mathcal{Q}_{3}=\{4\}$ but $\mathcal{Q}_{2}=\mathcal{Q}_{4}=\emptyset$.

At each time slot $i$, let $X_{i, \mathcal{C}_{k}}$ denote the set of observations in $X_{i,[1, M]}$ that come from the nodes in the $k$-th clique. Let $X_{i, \mathcal{S}_{k}}$ denote the observations in $X_{i,[1, M]}$ that come from the nodes in the $k$-th separator set. For any DGM, from the fact that the ordered sequence of cliques $\mathcal{C}_{1}, \mathcal{C}_{2}, \ldots, \mathcal{C}_{K}$ forms a perfect sequence, the joint distribution of $X_{i,[1, M]}$ follows the factorization $[22]^{2}$

$$
f\left(X_{i,[1, M]}\right)=\frac{\prod_{k=1}^{K} f\left(X_{i, \mathcal{C}_{k}}\right)}{\prod_{k=2}^{K} f\left(X_{i, \mathcal{S}_{k}}\right)}
$$

where $f(X)$ denotes the marginal pdf of $X$. Note that 15 can be derived using the pairwise Markov property in (4).

\section{Distributed Computation of the Generalized LiKelihood RATIO METHOd IN DGM}

In this section, we begin by reviewing the generalized loglikelihood ratio (GLR) procedure in the QCD problem [4]. The QCD problem can be modeled as a hypothesis testing problem, given by

$$
\begin{aligned}
& H_{0} \text { : no change occurs } \\
& H_{1} \text { : change occurs at a finite unknown time slot } \tau .
\end{aligned}
$$

Note that when the change occurs, all sensors are assumed to be affected simultaneously as mentioned in Assumption 1 .

The GLR test statistic up to the current time slot $n$ for 16 is

$$
\begin{aligned}
& G L R_{n}=\max \{0 \\
& \left.\max _{1 \leq n^{\prime} \leq n} \log \frac{\prod_{i=1}^{n^{\prime}-1} f_{0}\left(X_{i,[1, M]}\right) \prod_{i=n^{\prime}}^{n} f_{1}\left(X_{i,[1, M]}\right)}{\prod_{i=1}^{n} f_{0}\left(X_{i,[1, M]}\right)}\right\} \\
& =\max \left\{0, \max _{1 \leq n^{\prime} \leq n} \sum_{i=n^{\prime}}^{n} \log \frac{f_{1}\left(X_{i,[1, M]}\right)}{f_{0}\left(X_{i,[1, M]}\right)}\right\}
\end{aligned}
$$

${ }^{2} X_{i,[1, M]}$ following a decomposable graph is only a sufficient condition to guarantee 15 . In other cases we can attempt to verify 15 directly. 
where $G L R_{n}=0$ implies the decision maker does not declare change up to the current time slot $n$ and will continue acquiring more observations. In the optimum centralized QCD approach, at each time slot $n$, each sensor sends its observation to the FC. After receiving the data from all sensors, the FC calculates (18) and compares it to a threshold to decide whether to declare a change or continue to collect observations. In particular, the GLR procedure will raise an alarm at the time given by [4]

$$
T_{G L R}(b)=\inf \left\{n \geq 1: G L R_{n} \geq b\right\}
$$

where the constant $b>0$ needs to be chosen properly to satisfy the false alarm constraint in (1). The procedure in (19) is also called the classical centralized CUSUM algorithm which is shown to be optimal for [3] in [23].

Next we introduce our distributed approach. We make the following additional assumptions throughout the paper.

Assumption 2: At each time slot $i$, we assume that $X_{i,[1, M]} \triangleq\left(X_{i, 1}, X_{i, 2}, \ldots, X_{i, M}\right)$ satisfies the pairwise Markov property in (4) with respect to a given decomposable undirected graph $\mathcal{G}=(\mathcal{V}, \mathcal{E})$.

Assumption 3: Nodes in the same clique are close so that the energy cost of intra-clique communications is negligible compared to that of communications between the cliques to the FC, so we focus on communications between the cliques and the FC.

Assumption 4: The sets $\left\{\mathcal{S}_{k}\right\}_{k=2}^{K}$ and $\left\{\mathcal{C}_{k}\right\}_{k=1}^{K}$ do not change throughout the detection process. We generalize this in Section IV-D

Next we develop our distributed computation approach. From (15), we have

$$
\begin{aligned}
& \log \frac{f_{1}\left(X_{i,[1, M]}\right)}{f_{0}\left(X_{i,[1, M]}\right)} \\
&= \log \frac{\prod_{k=1}^{K} f_{1}\left(X_{i, \mathcal{C}_{k}}\right)}{\prod_{k=2}^{K} f_{1}\left(X_{i, \mathcal{S}_{k}}\right)} \prod_{k=1}^{K} f_{0}\left(X_{i, \mathcal{S}_{k}}\right) \\
&= \sum_{k=1}^{K} \log \frac{f_{1}\left(X_{i, \mathcal{C}_{k}}\right)}{f_{0}\left(X_{i, \mathcal{C}_{k}}\right)}-\sum_{k=2}^{K} \log \frac{f_{1}\left(X_{i, \mathcal{S}_{k}}\right)}{f_{0}\left(X_{i, \mathcal{S}_{k}}\right)} \\
&= \log \frac{f_{1}\left(X_{i, \mathcal{C}_{1}}\right)}{f_{0}\left(X_{i, \mathcal{C}_{1}}\right)}-\sum_{k \in \mathcal{Q}_{1}} \beta_{k} \log \frac{f_{1}\left(X_{i, \mathcal{S}_{k}}\right)}{f_{0}\left(X_{i, \mathcal{S}_{k}}\right)} \\
&+\sum_{j=2}^{K}\left(\log \frac{f_{1}\left(X_{i, \mathcal{C}_{j}}\right)}{f_{0}\left(X_{i, \mathcal{C}_{j}}\right)}-\alpha_{j} \log \frac{f_{1}\left(X_{i, \mathcal{S}_{j}}\right)}{f_{0}\left(X_{i, \mathcal{S}_{j}}\right)}\right. \\
&\left.\quad-\sum_{k \in \mathcal{Q}_{j}} \beta_{k} \log \frac{f_{1}\left(X_{i, \mathcal{S}_{k}}\right)}{f_{0}\left(X_{i, \mathcal{S}_{k}}\right)}\right) \\
&=\sum_{k=1}^{K} L_{k}\left(X_{i, \mathcal{C}_{k}}\right)
\end{aligned}
$$

where $\mathcal{Q}_{j}$ is defined in (11), and the set of non-negative coefficient pairs $\left\{\left(\alpha_{k}, \beta_{k}\right)\right\}_{k=2}^{K}$ satisfies

$$
\alpha_{k}+\beta_{k}=1, \forall k=2,3, \ldots, K \text {. }
$$

Note that 23 expresses $\log f_{1}\left(X_{i,[1, M]}\right) / f_{0}\left(X_{i,[1, M]}\right)$ as a sum of the clique statistics $L_{k}\left(X_{i, \mathcal{C}_{k}}\right)$ for $k=1,2, \ldots, K$ that are computed at each clique. After $[15]$ is used to obtain 20, we can group the separator terms into the associated clique terms based on the results in (8) and (9). In fact, each separator is a member of several cliques. For any term involving data coming from the $k$-th separator set, we can allocate $\alpha_{k}$ percentage of that term to the $k$-th clique and $\beta_{k}$ percentage to the other cliques that also contain the $k$ th separator set. This allows us to obtain (22) from (21). The centralized change detection test statistic can always be expressed as the sum in (23) as long as (24) is satisfied. From 24 , there are uncountably many choices of $\left\{\alpha_{k}, \beta_{k}\right\}_{k=2}^{K}$ which introduces flexibility in the definition of $L_{k}\left(X_{i, \mathcal{C}_{k}}\right)$ in 23) while still ensuring local computation. In 23, $L_{k}\left(X_{i, \mathcal{C}_{k}}\right)$ is defined as

$$
L_{1}\left(X_{i, \mathcal{C}_{1}}\right) \triangleq \log \frac{f_{1}\left(X_{i, \mathcal{C}_{1}}\right)}{f_{0}\left(X_{i, \mathcal{C}_{1}}\right)}-\sum_{k \in \mathcal{Q}_{1}} \beta_{k} \log \frac{f_{1}\left(X_{i, \mathcal{S}_{k}}\right)}{f_{0}\left(X_{i, \mathcal{S}_{k}}\right)}
$$

and for all $j=2,3, \ldots, K$,

$$
\begin{gathered}
L_{j}\left(X_{i, \mathcal{C}_{j}}\right) \triangleq \log \frac{f_{1}\left(X_{i, \mathcal{C}_{j}}\right)}{f_{0}\left(X_{i, \mathcal{C}_{j}}\right)}-\alpha_{j} \log \frac{f_{1}\left(X_{i, \mathcal{S}_{j}}\right)}{f_{0}\left(X_{i, \mathcal{S}_{j}}\right)} \\
-\sum_{k \in \mathcal{Q}_{j}} \beta_{k} \log \frac{f_{1}\left(X_{i, \mathcal{S}_{k}}\right)}{f_{0}\left(X_{i, \mathcal{S}_{k}}\right)} .
\end{gathered}
$$

Plugging (23) and (18) into (19) implies that the FC declares a change at time

$$
T_{C S}(b)=\inf \left\{n \geq 1: W_{n} \geq b\right\}
$$

where the CUSUM statistic $W_{n}$ is defined as

$$
W_{n} \triangleq \max \left\{0, \quad \max _{1 \leq n^{\prime} \leq n} \sum_{i=n^{\prime}}^{n} \sum_{k=1}^{K} L_{k}\left(X_{i, \mathcal{C}_{k}}\right)\right\} .
$$

A nice property of the non-negative CUSUM statistic $W_{n}$ is that it can be computed recursively as

$$
W_{n}=\max \left\{0, \quad W_{n-1}+\sum_{k=1}^{K} L_{k}\left(X_{n, \mathcal{C}_{k}}\right)\right\}
$$

with $W_{0}=0$. The above recursion is very useful because it requires little memory and is easily updated sequentially. Instead of directly sending all sensor observations to the FC, the distributed computation method provides the proper way to partition the sensor nodes into $K$ local groups that correspond to the cliques. Each clique $k$ will collect the information from the clique nodes to produce the clique statistic $L_{k}\left(X_{n, \mathcal{C}_{k}}\right)$ using 25 or 26 and then transmit it to the FC. The FC will compute the CUSUM statistic $W_{n}$ in 29) and compare it to the threshold $b$ to decide whether to raise an alarm or continue the process. We note that when we employ 27] with $W_{0}=0, \operatorname{WADD}\left(n^{\prime}\right)$ in 2] is equal to [3]

$$
\operatorname{WADD}\left(n^{\prime}\right)=\mathbb{E}_{1}\left[n^{\prime}-1\right]
$$

which implies that the worst case detection delay occurs at $\tau=1$. The result in 30 makes the computation of the WADD in (2) straightforward.

While the distributed computation method takes advantage 
of the graph structure to aggregate the statistics and avoids unnecessary long range transmissions, it is also of interest to further reduce the number of transmissions by the cliques to the FC. This is addressed in the next section.

\section{ENERGY-EFFICIENT QCD USING ORDERED TRANSMISSIONS}

In the last section the proposed distributed computation method implements the optimum centralized CUSUM algorithm while taking advantage of the graph structure using cliques. In order to further reduce the number of long distance transmissions from the cliques to the FC, in this section we describe an ordered transmission approach, and a lower bound on the average number of transmissions saved is provided. The savings are shown to be large for cases of interest.

\section{A. Ordered Transmissions for $Q C D$}

The idea of ordered transmissions for QCD is to order and then adaptively halt the transmissions of the clique statistics $\left\{L_{k}\left(X_{n, \mathcal{C}_{k}}\right)\right\}_{k=1}^{K}$ during each time slot $n$. Specifically, after grouping the nodes into several cliques, the clique with the largest clique statistic magnitude transmits first and the cliques with smaller clique statistic magnitudes possibly transmit later. This process is repeated during each time slot. We will show that by sometimes halting transmissions before all $K$ cliques have communicated their clique statistics, further transmissions can be saved while achieving the same detection delay as the optimal centralized CUSUM algorithm that requires all nodes to communicate their observations to the FC.

Our approach, which we call ordered-CUSUM, is summarized in Algorithm 1. At the beginning of the current time slot $n$ (denoted time $t_{n}$ ) each clique $k$ for $k=1, \ldots, K$ determines a time $t_{n, k}=t_{n}+\eta /\left|L_{k}\left(X_{n, \mathcal{C}_{k}}\right)\right|$ to transmit its local statistic $L_{k}\left(X_{n, \mathcal{C}_{k}}\right)$ to the $\mathrm{FC}$, where the positive number $\eta$ can be made as small as the system will allow. Thus clique transmissions are time ordered using $t_{n}<t_{n, k_{1}} \leq t_{n, k_{2}} \leq$ $\ldots \leq t_{n, k_{K}}<t_{n+1}$ where $k_{j}$ is the index of the clique which has the $k_{j}$-th largest $\left|\hat{L}_{n, k_{j}}\right|$ such that

$$
\left|\hat{L}_{n, 1}\right| \geq\left|\hat{L}_{n, 2}\right| \geq \ldots \geq\left|\hat{L}_{n, K}\right| \text {. }
$$

In this way the cliques with larger-in-magnitude local test statistic transmit earlier. When the FC receives a new transmission from a clique $k_{j}$, it computes

$$
W_{n, k_{j}} \triangleq W_{n-1}+\sum_{k=1}^{k_{j}} \hat{L}_{n, k}
$$

where $W_{n-1}$ is the CUSUM statistic at time slot $(n-1)$, and compares $W_{n, k_{j}}$ from 32 with the updated threshold

$$
\phi_{n, L} \triangleq-\left(K-k_{j}\right)\left|\hat{L}_{n, k_{j}}\right| \text {. }
$$

By sending a message to all cliques, the FC stops any further clique transmission when $W_{n, k_{j}} \leq \phi_{n, L}$. When this occurs the FC declares $W_{n}=0$ and the system progresses to the next

\footnotetext{
${ }^{3}$ In practice it may be desired to stop at some large value of time slot $n$, even if $W_{n} \geq b$ has not been satisfied.
}

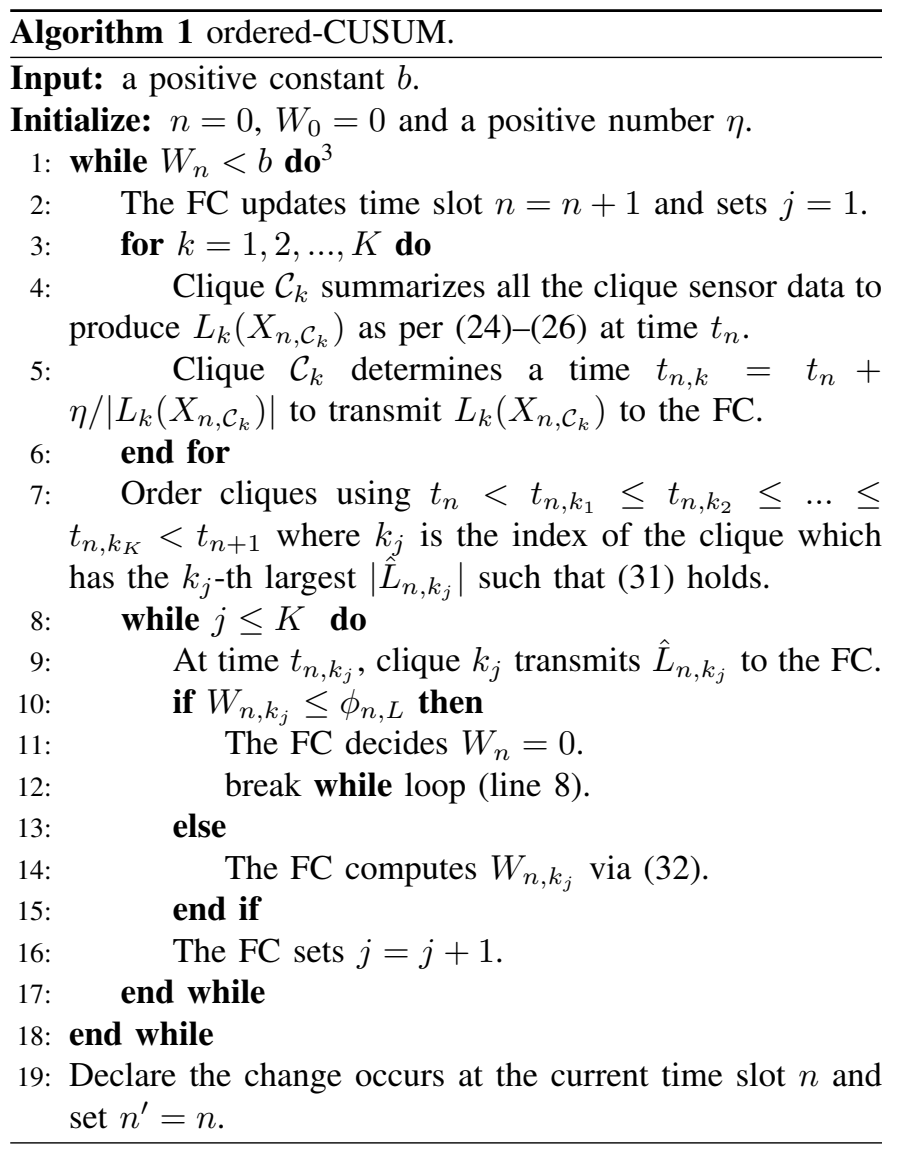

time slot $t_{n+1}$. If all cliques transmit prior to $W_{n, k_{j}} \leq \phi_{n, L}$, then the current time slot is also ended and a decision is made using (27) and (32). If all transmission propagation delays are known and timing is synchronized, one can schedule all transmissions back to the FC so they arrive in the correct order. However, the process can easily be implemented with robustness to small timing errors. Even with inaccurate estimates of propagation delays or imperfect synchronization, since the FC receives the values to be ordered, the FC can put them back in order correctly as long as the FC waits a short period related to the uncertainty. By design, the ordered-CUSUM algorithm will always be optimal, as summarized in the following Theorem.

Theorem 1: Consider the QCD problem defined in (3). At each time slot $n<\tau$, ordered-CUSUM summarized in Algorithm 1 achieves the same detection performance as the optimum centralized approach while using a smaller average number of transmissions.

Proof: In the ordered-CUSUM algorithm, during time slot $n$, when the FC receives a new clique statistic it updates the threshold $\phi_{n, L}$ via (33), and compares $\phi_{n, L}$ with $W_{n, k^{\prime}}$ in 32. Let the most recent transmission be given by $\hat{L}_{n, k_{j}}$. Due to ordering it follows that $\left|\hat{L}_{n, k_{j}}\right|$ is an upper bound for those of the clique statistics which have not yet been transmitted. It further follows that the sum of the clique statistics that have not yet transmitted must be less than or equal to $\left(K-k_{j}\right)\left|\hat{L}_{n, k_{j}}\right|$, which is equal to $-\phi_{n, L}$. If $W_{n, k_{j}} \leq \phi_{n, L}$, then $W_{n}$ has to be zero according to (29), regardless of the clique statistics that have not yet been transmitted. Hence, even without receiving further transmissions, the FC can implement the optimum cen- 
tralized communication unconstrained approach and declare $W_{n}=0$ at time slot $n$. On the other hand, the optimum centralized communication unconstrained approach continues to transmit at time slot $n$ with nonzero probability. Thus, at each time slot $n$, the average number of transmissions required by ordered-CUSUM is smaller than that of the optimum centralized communication unconstrained approach while the detection performance is the same.

While we have shown that the ordered-CUSUM algorithm built on ordering is more communication-efficient than the optimum centralized communication unconstrained approach, it is interesting to consider whether there exists a lower bound on the average number of transmissions saved by the orderedCUSUM algorithm. This question will be addressed in the next subsection.

\section{B. Lower Bound on the Average Number of Transmissions Saved}

In this subsection, we derive a lower bound on the average number of transmissions saved by ordered-CUSUM. The following theorem formally describes the communication saving lower bound for each time slot $n$.

Theorem 2: Consider the QCD problem described in (3). When using ordered-CUSUM, for any choice of the pairs $\left\{\left(\alpha_{k}, \beta_{k}\right)\right\}_{k=2}^{K}$ with $K \geq 2$ and $\alpha_{k}+\beta_{k}=1$, the average number of transmissions saved $S_{n}$ for time slot $n$ is bounded from below by

$$
S_{n}>\left(\left\lceil\frac{K}{2}\right\rceil-1\right) \operatorname{Pr}\left(W_{n-1}=0 \text { and } L_{k}\left(X_{n, \mathcal{C}_{k}}\right)<0, \forall k\right) \text {. }
$$

Proof: According to ordered-CUSUM, if $W_{n, k_{j}}$ defined in (32) is smaller than the threshold $\phi_{n, L}$ in 33 , then transmissions will be stopped during time slot $n$ and the algorithm proceeds to the next time slot $(n+1)$. Let

$$
\begin{aligned}
k_{n}^{*} \triangleq \min \left\{1 \leq k^{\prime}\right. & <K: W_{n-1}+\sum_{k=1}^{k^{\prime}} \hat{L}_{n, k} \\
& \left.<-\left(K-k^{\prime}\right)\left|\hat{L}_{n, k^{\prime}}\right|\right\}
\end{aligned}
$$

denote the number of necessary transmissions during time slot $n$ using ordered-CUSUM, and define

$$
S_{n} \triangleq \mathbb{E}\left[K-k_{n}^{*}\right]
$$

as the average number of transmissions saved at time slot $n$. Then from (36), $S_{n}$ can be bounded from below by

$$
\begin{aligned}
S_{n} & =\sum_{k=1}^{K}(K-k) \operatorname{Pr}\left(k_{n}^{*}=k\right) \\
& \geq \sum_{k=1}^{\lfloor K / 2\rfloor+1}(K-k) \operatorname{Pr}\left(k_{n}^{*}=k\right) \\
& >\left(\left\lceil\frac{K}{2}\right\rceil-1\right) \sum_{k=1}^{\lfloor K / 2\rfloor+1} \operatorname{Pr}\left(k_{n}^{*}=k\right) \\
& =\left(\left\lceil\frac{K}{2}\right\rceil-1\right) \operatorname{Pr}\left(k_{n}^{*} \leq\left\lfloor\frac{K}{2}\right\rfloor+1\right) .
\end{aligned}
$$

The result in (38) is obtained by dropping some non-negative terms in (37). In going from (38) to 39, we bound $(K-k)$ by using $(\lceil K / 2\rceil-1)$ for $k=1, \ldots,\lfloor K / 2\rfloor+1$. Plugging the definition of $k_{n}^{*}$ from (35) into 40, we obtain

$$
\begin{aligned}
S_{n}>\left(\left\lceil\frac{K}{2}\right\rceil-1\right) \operatorname{Pr}\left(W_{n-1}+\sum_{k=1}^{\lfloor K / 2\rfloor+1} \hat{L}_{n, k}\right. \\
\left.\leq-\left(\left\lceil\frac{K}{2}\right\rceil-1\right)\left|\hat{L}_{n,\left\lfloor\frac{K}{2}\right\rfloor+1}\right|\right) \\
\geq\left(\left\lceil\frac{K}{2}\right\rceil-1\right) \operatorname{Pr}\left(W_{n-1}=0, \hat{L}_{n, k}<0, \forall k,\right. \text { and } \\
W_{n-1}+\sum_{k=1}^{\lfloor K / 2\rfloor+1} \hat{L}_{n, k} \leq-\left(\left\lceil\frac{K}{2}\right\rceil-1\right) \mid \hat{L}_{\left.n,\left\lfloor\frac{K}{2}\right\rfloor+1 \mid\right)} \\
\geq\left(\left\lceil\frac{K}{2}\right\rceil-1\right) \operatorname{Pr}\left(W_{n-1}=0 \text { and } \hat{L}_{n, k}<0, \forall k\right) \\
=\left(\left\lceil\frac{K}{2}\right\rceil-1\right) \operatorname{Pr}\left(W_{n-1}=0 \text { and } L_{k}\left(X_{n, \mathcal{C}_{k}}\right)<0, \forall k\right) .
\end{aligned}
$$

In going from (41) to 42, we add two extra constraints which will maintain or reduce the probability. In 42, when $W_{n-1}=$ 0 and $\hat{L}_{n, k}<0, \forall k$ are true, $W_{n-1}+\sum_{k=1}^{\lfloor K / 2\rfloor+1} \hat{L}_{n, k} \leq$ $-(\lceil K / 2\rceil-1)\left|\hat{L}_{n,\lfloor K / 2\rfloor+1}\right|$ must be true which implies the result in (43). In going from (43) to (44), we use the fact that all of the ordered statistics $\hat{L}_{n, k}$ being negative implies all of the original unordered statistics $L_{k}\left(X_{n, \mathcal{C}_{k}}\right)$ are negative. This completes the proof.

We point out that the lower bound in (44) is very general and is valid for any DGM and any choice of the set of nonnegative coefficient pairs $\left\{\left(\alpha_{k}, \beta_{k}\right)\right\}_{k=2}^{K}$ with $\alpha_{k}+\beta_{k}=1$. The result in 44 indicates that the lower bound on $S_{n}$ depends on the number of cliques and the joint statistics of $W_{n-1}$ and $L_{k}\left(X_{n, \mathcal{C}_{k}}\right)$. In the next subsection we show that the savings can be large for several cases of interest.

\section{Large Saving Gains for Several Cases of Interest}

Consider a distance measure $s$ between the distributions of the sensor observations before and after the change time $\tau$. The distance measure $s$ is assumed to satisfy the following mild condition.

Assumption 5: For the hypothesis testing problem considered in (16) with $L_{k}\left(X_{n, \mathcal{C}_{k}}\right)$ as per 24)-26), we assume that the probability $\operatorname{Pr}\left(L_{k}\left(X_{n, \mathcal{C}_{k}}\right)<0\right) \rightarrow 1$ as $s \rightarrow \infty$ for all $k=1, \ldots, K$ and $n<\tau$.

Intuitively, with a large distance between the distributions of the sensor observations before and after the change time, it should be easy for the FC to decide when the change occurs. At the end of this subsection, we provide two popular general QCD problems and the corresponding distance measure to illustrate that Assumption 5 is reasonable.

Under Assumption 5, the following theorem describes the limiting behavior of the lower bound on the total number of transmissions saved by ordered-CUSUM. 
Theorem 3: Under Assumptions 17-5, consider the approach in Algorithm 1 for the QCD problem in (3). With a sufficiently large $s$, the total number of transmissions saved over the optimum centralized communication unconstrained approach increases at least as fast as proportional to $K$ while the detection delay is not affected. In particular, the total number of transmissions saved is lower bounded by $(\lceil K / 2\rceil-1)(\tau-1)$.

Proof: From (34) in Theorem 2, we have

$S_{n}>\left(\left\lceil\frac{K}{2}\right\rceil-1\right) \operatorname{Pr}\left(W_{n-1}=0\right.$ and $\left.L_{k}\left(X_{n, \mathcal{C}_{k}}\right)<0, \forall k\right)$

Next we employ induction to show that as $s \rightarrow \infty$, for $n<\tau$, we have

$$
\operatorname{Pr}\left(W_{n-1}=0 \text { and } L_{k}\left(X_{n, \mathcal{C}_{k}}\right)<0, \forall k\right) \rightarrow 1 .
$$

Throughout this proof, we only consider the time slots before the change occurs which means we focus on the case when $n<\tau$. Specifically, we set $W_{0}=0$. For a sufficiently large $s$, Assumption 5 implies that all clique statistics are negative for $n<\tau$. Thus, the probability $\operatorname{Pr}\left(W_{0}=0\right.$ and $L_{k}\left(X_{1, \mathcal{C}_{k}}\right)<$ $0, \forall k) \rightarrow 1$ implies $\operatorname{Pr}\left(W_{1}=0\right) \rightarrow 1$. If we assume $\operatorname{Pr}\left(W_{n-2}=0\right) \rightarrow 1$ at time slot $(n-1)$, then under Assumption 5 we have $\operatorname{Pr}\left(W_{n-2}=0\right.$ and $\left.L_{k}\left(X_{n-1, \mathcal{C}_{k}}\right)<0, \forall k\right) \rightarrow 1$ for $n<\tau$ which implies $\operatorname{Pr}\left(W_{n-1}=0\right) \rightarrow 1$. Thus, at time slot $n$, we obtain $\operatorname{Pr}\left(W_{n-1}=0\right.$ and $\left.L_{k}\left(X_{n, \mathcal{C}_{k}}\right)<0, \forall k\right) \rightarrow 1$ for $n<\tau$. From (45), for all $n<\tau$, we have

$$
S_{n}>\left\lceil\frac{K}{2}\right\rceil-1 \text {. }
$$

Finally, it follows that the total number of transmissions saved $K_{s}$ can be bounded by

$$
K_{s}>\left(\left\lceil\frac{K}{2}\right\rceil-1\right)(\tau-1) .
$$

As illustrated by Theorem 3 , the total number of transmissions saved by ordering the communications from the cliques to the FC increases at least as fast as linearly proportional to the number of cliques $\mathrm{K}$ while achieving the same detection delay as the optimum centralized communication unconstrained approach. Theorem 3 also states that more transmissions can be saved as the change time increases. In the following, we provide two general problems and the corresponding distance measure.

Example 1: Consider detecting a change in the mean of a sequence of sensor observation vectors following a multivariate Gaussian distribution as 4

$$
\begin{aligned}
& X_{n,[1, M]} \sim \mathcal{N}(\mathbf{0}, \boldsymbol{\Sigma}) \text { when } n<\tau \\
& X_{n,[1, M]} \sim \mathcal{N}(\boldsymbol{\mu}, \boldsymbol{\Sigma}) \text { when } n \geq \tau
\end{aligned}
$$

where $\boldsymbol{\mu} \neq \mathbf{0}$ and the known covariance matrix $\boldsymbol{\Sigma}$ is assumed to be positive definite. In this problem, we can choose $s=$ $\min _{k}\left\|\boldsymbol{\mu}_{\mathcal{C}_{k}}\right\|$ as the distance measure where $\boldsymbol{\mu}_{\mathcal{C}_{k}}$ denotes the mean vector of the nodes in the $k$-th clique with $\ell_{2}$-norm $\left\|\boldsymbol{\mu}_{\mathcal{C}_{k}}\right\|$.

\footnotetext{
${ }^{4} \mathcal{N}(\mathbf{a}, \mathbf{A})$ denotes the multivariate Gaussian distribution with mean $\mathbf{a}$ and covariance matrix $\mathbf{A}$.
}

Example 2: Consider detecting a change in the covariance matrix of a sequence of sensor observation vectors following a multivariate Gaussian distribution as

$$
\begin{aligned}
& X_{n,[1, M]} \sim \mathcal{N}(\mathbf{0}, \mathbf{I}) \text { when } n<\tau \\
& X_{n,[1, M]} \sim \mathcal{N}(\mathbf{0}, \mathbf{\Sigma}) \text { when } n \geq \tau,
\end{aligned}
$$

where $\mathbf{I}$ is an identity matrix and the known covariance matrix $\boldsymbol{\Sigma}$ is assumed to be positive definite. In this problem, $s=$ $\min _{k} \lambda_{\min , k}$ where $\lambda_{\min , k}$ is the minimum eigenvalue of $\boldsymbol{\Sigma}_{\mathcal{C}_{k}}$ which denotes the covariance matrix associated with $X_{n, \mathcal{C}_{k}}$ for $n \geq \tau$. The following theorem shows that Assumption 5 is valid for Examples 1 and 2

Theorem 4: Consider the problems in Example 1 and Example 2 and the ordered transmission approach described in the ordered-CUSUM algorithm which employs (23) with

$$
\alpha_{k}=1-2^{K-k} \xi,
$$

and

$$
\beta_{k}=2^{K-k} \xi
$$

for all $k=2,3, \ldots, K$ using any $\xi$ which satisfies

$$
\xi \in\left(0, \frac{1}{2^{K-1}-1}\right) \text {. }
$$

For any $k=1,2, \ldots, K$ with $K>2$, with sufficiently large $\min _{k}\left\|\boldsymbol{\mu}_{\mathcal{C}_{k}}\right\|$ for 49 ) or sufficiently large $\min _{k} \lambda_{\min , k}$ for 50 , we have for all $k=1,2, \ldots, K$ and $n<\tau$,

$$
\operatorname{Pr}\left(L_{k}\left(X_{n, \mathcal{C}_{k}}\right)<0\right) \rightarrow 1
$$

Proof: The proof of this theorem is omitted, since it follows from the proof in [15] and [16]. Specifically, for the problem in (49), as $s \rightarrow \infty$ with $s=\min _{k}\left\|\boldsymbol{\mu}_{\mathcal{C}_{k}}\right\|$, the result in (54) can be obtained by following Theorem 2 in [15]. For the problem in (50), as $s \rightarrow \infty$ with $s=\min _{k} \lambda_{\min , k}$, then (54) can be obtained by following Theorem 3 in [16].

\section{Extensions to the Case Where the Graph Structure Changes.}

In this subsection, we generalize Assumption 4 and consider the case where the graph is fixed under each hypothesis but the graph structure of $f_{0}\left(X_{n,[1, M]}\right)$ (relevant for $\left.n<\tau\right)$ is not the same as $f_{1}\left(X_{n,[1, M]}\right)$ (relevant for $\left.n \geq \tau\right)$. Suppose the sensor observations $X_{n,[1, M]}$ obey the pairwise Markov property with respect to a decomposable graph $\mathcal{G}_{1}=\left(\mathcal{V}_{1}, \mathcal{E}_{1}\right)$ before the change $(n<\tau)$ and a decomposable graph $\mathcal{G}_{2}=\left(\mathcal{V}_{2}, \mathcal{E}_{2}\right)$ after the change $(n \geq \tau)$ with $\mathcal{G}_{1} \neq \mathcal{G}_{2}$. In order to implement distributed computation and ordered transmissions for this case, the sequence of cliques $\left\{\mathcal{C}_{k}\right\}_{k=1}^{K}$ is derived based on $\mathcal{G}=\mathcal{G}_{1} \cup \mathcal{G}_{2}$ instead of $\mathcal{G}_{1}$ or $\mathcal{G}_{2}$. Compared to $\mathcal{G}_{1}$ and $\mathcal{G}_{2}$, the graph structure $\mathcal{G}$ possibly increases the size of some cliques, implying extra node data needs to be collected in these larger cliques. However, when we employ the known pdf $f_{0}\left(X_{n,[1, M]}\right)$ or $f_{1}\left(X_{n,[1, M]}\right)$ in these computations, we use some of the extra data in computations involving $f_{0}\left(X_{n,[1, M]}\right)$ and the rest in computations involving $f_{1}\left(X_{n,[1, M]}\right)$. Thus, the graph $\mathcal{G}$ allows the computations that either $\mathcal{G}_{1}$ or $\mathcal{G}_{2}$ require.

Consider the example illustrated in Fig. 2. Before the change, the graph structure is indicated by graph $\mathcal{G}_{1}$ which 


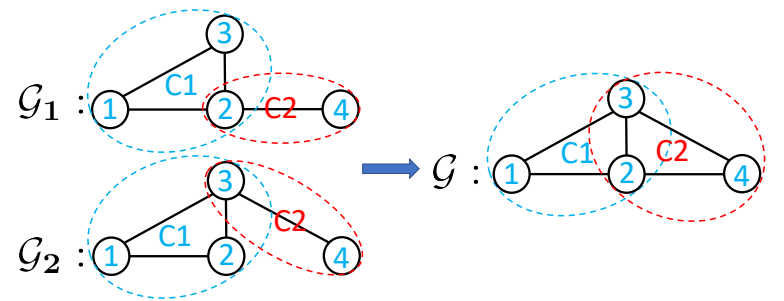

Fig. 2. Choice of cliques and separator sets when graph structure changes.

has two clique sets $\mathcal{C}_{1}=\{1,2,3\}$ and $\mathcal{C}_{2}=\{2,4\}$ along with one separator set $\mathcal{S}_{2}=\{2\}$. After the change occurs, the graph structure illustrated in $\mathcal{G}_{2}$ has two clique sets $\mathcal{C}_{1}=\{1,2,3\}$ and $\mathcal{C}_{2}=\{3,4\}$ along with the separator set $\mathcal{S}_{2}=\{3\}$. In order to keep the clique and separator sets the same through the detection process, we collect nodes into the cliques of the graph $\mathcal{G}=\mathcal{G}_{1} \cup \mathcal{G}_{2}$ whose clique sets are $\mathcal{C}_{1}=\{1,2,3\}$ and $\mathcal{C}_{2}=\{2,3,4\}$. We also employ the separator set $\mathcal{S}_{2}=\{2,3\}$. These sets are respectively regarded as the clique sets and the separator set through the whole detection process. Note that compared to $\mathcal{C}_{2}=\{2,4\}$ in $\mathcal{G}_{1}, \mathcal{G}$ defines a new larger clique $\mathcal{C}_{2}=\{2,3,4\}$ to indicate that the data at node 3 will be collected at clique $\mathcal{C}_{2}$. However, when we compute $f_{0}\left(X_{n,[1,4]}\right)$ in a distributed way, we do not really use the data from node 3 in $\mathcal{C}_{2}$ to compute $f_{0}\left(X_{n, \mathcal{C}_{2}}\right)$. Similarly, when we compute $f_{1}\left(X_{n,[1,4]}\right)$, we do not really use the data from node 2 in $\mathcal{C}_{2}$ to compute $f_{1}\left(X_{n, \mathcal{C}_{2}}\right)$. After implementing the distributed computation using the clique and separator sets of $\mathcal{G}$, ordered transmissions can be developed according to Section IV] It is worth mentioning that the union operation might decrease the number of cliques which can degrade the gains of distributed processing and ordering.

\section{Numerical RESUlts}

In this section, numerical examples for two representative classes of decomposable graphical models (chain structure and tree structure) are presented in order to illustrate the communication saving performance using the proposed ordered transmission approach. Chain structure and tree structure graphs have been employed in studies on feature representation [24], topology identification [25], structure learning [26] and electrical power systems [21].

\section{A. Total number of Transmissions Saved versus the Distance Measure}

In this subsection, the lower bound in (34) is compared with the actual number of transmissions saved by ordered-CUSUM from Monte Carlo simulations (1000 runs). Consider a graph with chain structure as illustrated in Fig. 3 where we set the number of cliques $K=50$. As indicated in Fig. 3. each clique has 3 nodes, and every two-connected clique pair are coupled through a 2 -sensor separator set. We first consider the change detection problem in (49) and generate a covariance matrix which satisfies the conditional independence specified by the graph structure in Fig. 3 In the simulation results of Fig. 3 . we set $\tau=1, \xi=0.5 /\left(2^{49}-1\right)$ and $\boldsymbol{\mu}=c[1,1, \ldots, 1]^{\top}$. In

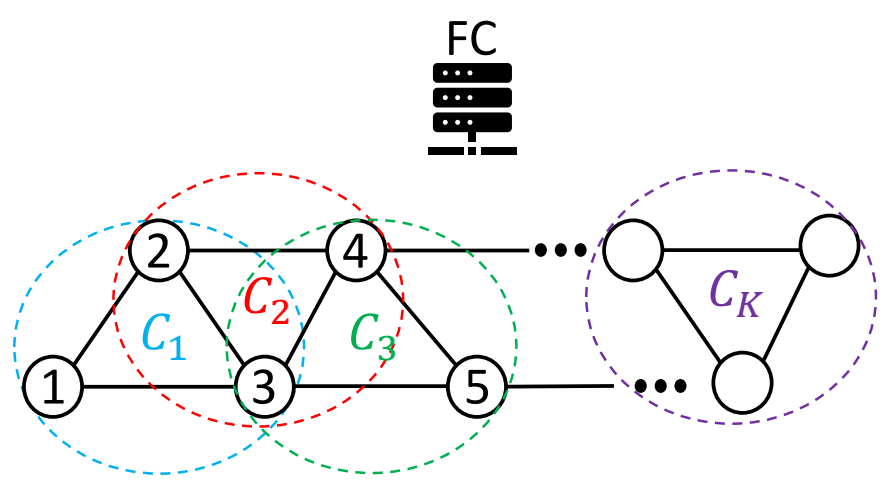

Fig. 3. The decomposable graphical model with chain structure. Clique 1: Nodes 1,2,3; Separator: Nodes 2,3; Clique 2: Nodes 2,3,4; Separator: Nodes 3,4 ; and so on down the chain. Each clique communicates with the fusion center (FC).

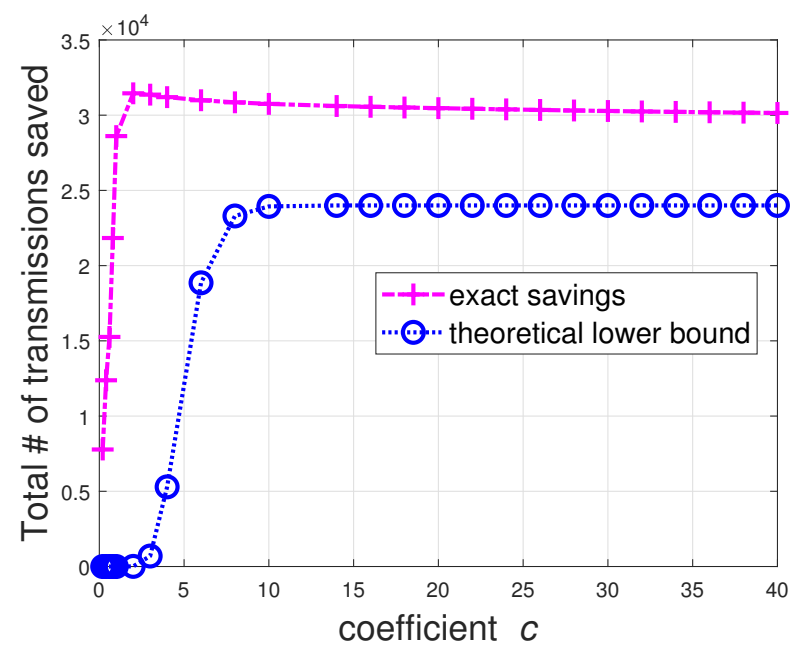

Fig. 4. Impact of mean shift on the total number of transmissions saved when the change does not occur during these $10^{3}$ time slots.

order to satisfy the false alarm constraint $\mathbb{E}_{\infty}(n) \geq \gamma=10^{3}$, the minimum value of the positive constant $b$ is found using grid search with the grid points spaced 0.01 apart. Note that hereafter in the simulation results, we only count the number of transmissions from the cliques to the FC for the first $10^{3}$ time slots. In Fig. 3, we plot the total number of transmissions saved versus $c$ when the change does not occur during these $10^{3}$ time slots. Fig. 3 indicates that our theoretical lower bound in 34 is valid and its value increases as $c$ increases which means the distance measure $s$ increases. As expected from our analysis, Fig. 3 shows that the lower bound on the total number of transmissions saved nearly equals 24000 when $c=$ 40 which is consistent with Theorem 3 since $(\lceil K / 2\rceil-1) \times$ $10^{3}=24000$ when $K=50$.

\section{B. Total Number of Transmissions Saved versus the Number of Cliques}

In this subsection, using Monte Carlo simulations (1000 runs), we investigate the total number of transmissions saved for the first $10^{3}$ time slots by ordering the communications from the cliques to the $\mathrm{FC}$ for different number of cliques 


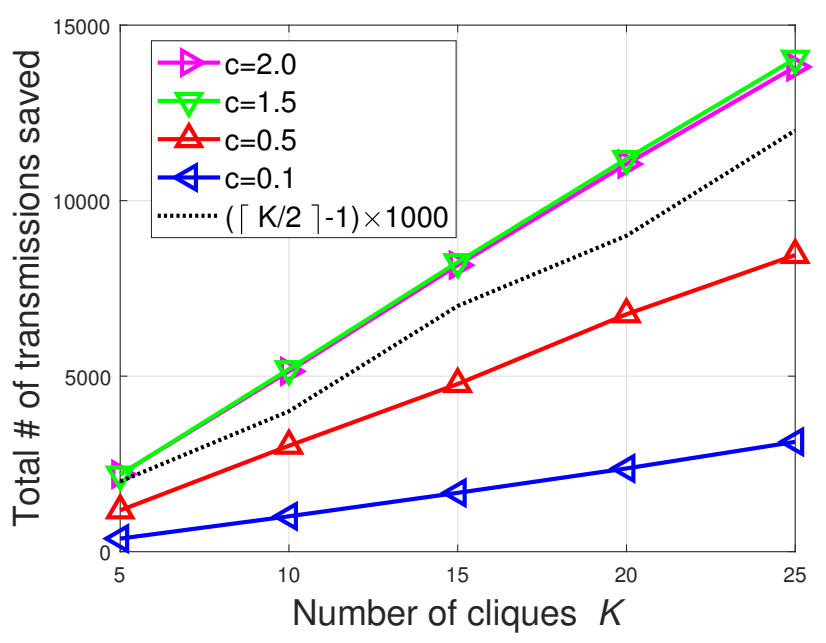

Fig. 5. The total number of transmissions saved when the change does not occur during these $10^{3}$ time slots versus $K$ for the model illustrated in Fig. 3

$K$ for the case when no change occurs during these $10^{3}$ time slots. We consider the testing problem in (49) with the same class of graph structures as in Fig. 3 . We plot the total number of transmissions saved when no change occurs during these $10^{3}$ time slots versus $K$ in Fig. 5 for the parameters $\tau=1, \gamma=10^{3}, \xi=0.5 /\left(2^{K-1}-1\right)$ and $\boldsymbol{\mu}=c[1,1, \ldots, 1]^{\top}$. For comparison, the limiting theoretical lower bound on communication savings in Theorem 3 is also provided. For the specific cases considered, Fig. 5 indicates that the total number of transmissions saved by OrderedCUSUM increases approximately linearly with $K$ for every value of $c$. It also indicates that the rate of increase with $K$ increases with increasing $c$ for smaller $c$ but eventually, the rate of increase saturates as $c$ becomes large, corresponding to a large and easily detectable change.

Next, we consider a different class of graphs with the tree structure as illustrated in Fig. 6 where each clique contains 4 nodes and every two-connected clique pair are coupled through a 1-sensor separator set. Here we consider the testing problem in 50. We set $\tau=1, \gamma=10^{3}$ and $\xi=0.5 /\left(2^{K-1}-1\right)$. The diagonal elements of $\Sigma_{\mathcal{C}_{k}}$ for all $k$ are set to be $x^{2}$ and the other elements of $\boldsymbol{\Sigma}_{\mathcal{C}_{k}}$ are set to equal to $x / 10$ where the minimum value of $\Sigma_{\mathcal{C}_{k}}$ is $x^{2}-x / 10$, so its value may be changed by varying $x$. In Fig. 7, we plot the total number of transmissions saved by ordered-CUSUM when the change does not occur during $10^{3}$ time slots versus $K$ for different values of $x$. Fig. 7 implies that the total number of transmissions saved increases approximately linearly with $K$ for every value of $x$. Fig. 7 also indicates that when $x$ is relatively small then increasing $x$ increases the slope which is very similar to the result in Fig. 5 .

\section{CONCLUSION}

In this paper a new class of communication-efficient QCD schemes for sensor networks have been developed that reduce the number of transmissions without any impact on detection delay when compared to the optimum centralized

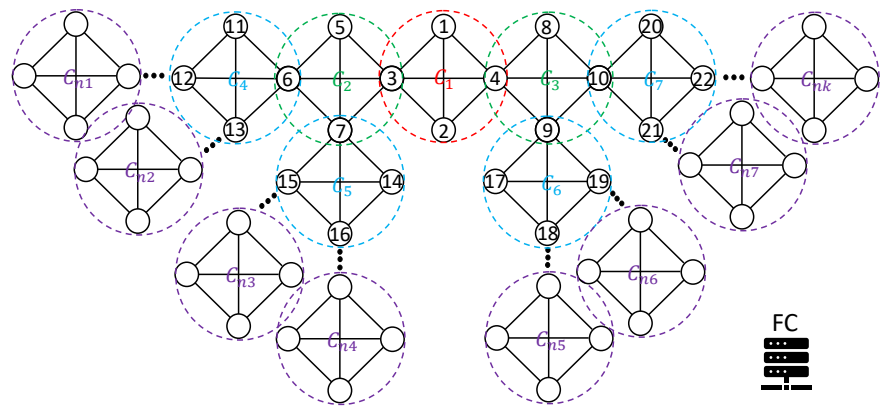

Fig. 6. The decomposable graphical model with tree structure. Clique 1: Nodes 1,2,3,4; Separator: Nodes 3,4; Clique 2: Nodes 3,5,6,7; Separator: Nodes 6,7; and so on. Each clique communicates with the fusion center (FC).

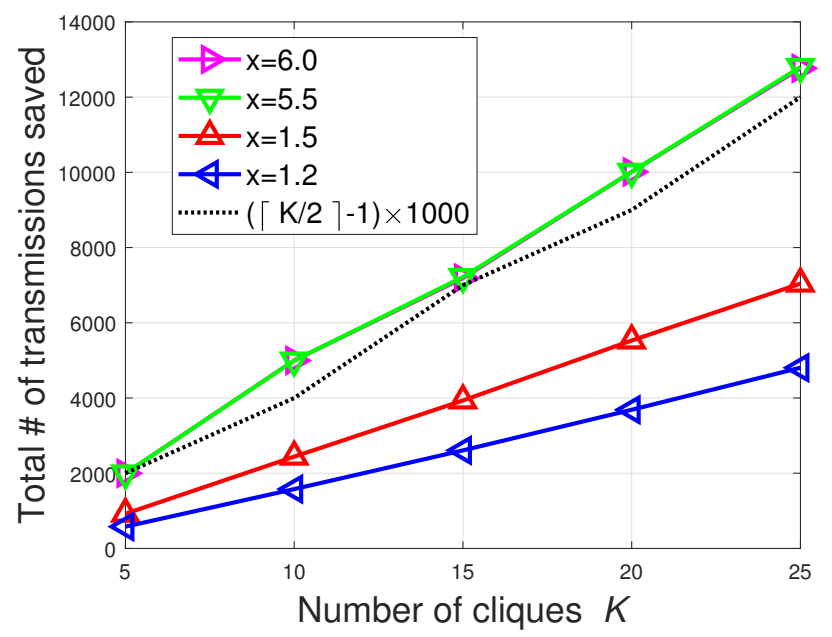

Fig. 7. The total number of transmissions saved when the change does not occur during these $10^{3}$ time slots versus $K$ for the model illustrated in Fig. 6

communication unconstrained QCD approach. It is assumed that the observations follow a decomposable graphical model (DGM), which is a very broad class of network topologies, and the observations between sensors may be dependent. For a QCD problem with sensor observations following any DGM, we write the optimum centralized change detection test statistic as a sum of clique statistics where each clique statistic can be computed only using local data available at the corresponding clique. To complete the computation of the optimum centralized test, each clique forwards its clique statistic to the FC.

In order to further improve the communication efficiency, we have applied the ordered transmission approach over the cliques to reduce the number of transmissions from the cliques to the FC without performance loss. In the ordered transmission approach, the cliques with more informative observations transmit their clique statistics to the FC first. Transmissions are halted after sufficient evidence is accumulated to save transmissions, and a new round of sensing is initiated. Furthermore, a lower bound on the average number of transmissions saved has been provided. When a well-behaved distance measure between the pdfs of the sensor observations before and after the change becomes sufficiently large, the lower bound approaches 
approximately half the number of cliques. Extensions to the case where the graph structure changes have been discussed. In order to illustrate our theoretical analysis, two popular general QCD problems with sensor observations following a multivariate Gaussian distribution have been considered and numerical results have been provided which are consistent with the analytical findings.

\section{REFERENCES}

[1] I. F. Akyildiz, W. Su, Y. Sankarasubramaniam, and E. Cayirci, "Wireless sensor networks: a survey," Computer networks, vol. 38, no. 4, pp. 393 422, 2002.

[2] C.-Y. Chong and S. P. Kumar, "Sensor networks: evolution, opportunities, and challenges," Proceedings of the IEEE, vol. 91, no. 8, pp. 1247-1256, 2003.

[3] V. V. Veeravalli and T. Banerjee, "Quickest change detection," in Academic Press Library in Signal Processing. Elsevier, 2014, vol. 3, pp. 209-255.

[4] Y. Mei, "Efficient scalable schemes for monitoring a large number of data streams," Biometrika, vol. 97, no. 2, pp. 419-433, 2010.

[5] T. Banerjee and V. V. Veeravalli, "Data-efficient quickest change detection in sensor networks," IEEE Transactions on Signal Processing, vol. 63, no. 14, pp. 3727-3735, 2015.

[6] G. Lorden et al., "Procedures for reacting to a change in distribution," The Annals of Mathematical Statistics, vol. 42, no. 6, pp. 1897-1908, 1971.

[7] T. L. Lai, "Information bounds and quick detection of parameter changes in stochastic systems," IEEE Transactions on Information Theory, vol. 44, no. 7, pp. 2917-2929, 1998.

[8] M. Pollak, "Optimal detection of a change in distribution," The Annals of Statistics, pp. 206-227, 1985.

[9] V. V. Veeravalli, "Decentralized quickest change detection," IEEE Transactions on Information theory, vol. 47, no. 4, pp. 1657-1665, 2001.

[10] Y. Mei, "Information bounds and quickest change detection in decentralized decision systems," IEEE Transactions on Information theory, vol. 51, no. 7, pp. 2669-2681, 2005.

[11] A. G. Tartakovsky and V. V. Veeravalli, "Asymptotically optimal quickest change detection in distributed sensor systems," Sequential Analysis, vol. 27, no. 4, pp. 441-475, 2008.

[12] C. Rago, P. Willett, and Y. Bar-Shalom, "Censoring sensors: A lowcommunication-rate scheme for distributed detection," IEEE Transactions on Aerospace and Electronic Systems, vol. 32, no. 2, pp. 554-568, 1996.

[13] Y. Mei, "Quickest detection in censoring sensor networks," in 2011 IEEE International Symposium on Information Theory Proceedings. IEEE, 2011, pp. 2148-2152.

[14] R. S. Blum and B. M. Sadler, "Energy efficient signal detection in sensor networks using ordered transmissions," IEEE Transactions on Signal Processing, vol. 56, no. 7, pp. 3229-3235, 2008.

[15] J. Zhang, Z. Chen, R. S. Blum, X. Lu, and W. Xu, "Ordering for reduced transmission energy detection in sensor networks testing a shift in the mean of a gaussian graphical model," IEEE Transactions on Signal Processing, vol. 65, no. 8, pp. 2178-2189, 2017.

[16] Y. Chen, R. S. Blum, B. M. Sadler, and J. Zhang, "Testing the structure of a gaussian graphical model with reduced transmissions in a distributed setting," IEEE Transactions on Signal Processing, vol. 67, no. 20, pp. 5391-5401, 2019.

[17] Y. Chen, R. Blum, and B. Sadler, "Optimal quickest change detection in sensor networks using ordered transmissions," in 2020 IEEE 21st International Workshop on Signal Processing Advances in Wireless Communications (SPAWC) (IEEE SPAWC 2020), Atlanta, USA, May 2020.

[18] Y. Xiang, S. K. M. Wong, and N. Cercone, "A microscopic study of minimum entropy search in learning decomposable markov networks," Machine Learning, vol. 26, no. 1, pp. 65-92, 1997.

[19] K. Mohan, P. London, M. Fazel, D. Witten, and S.-I. Lee, "Node-based learning of multiple gaussian graphical models," The Journal of Machine Learning Research, vol. 15, no. 1, pp. 445-488, 2014.

[20] M. Cetin, L. Chen, J. W. Fisher, A. T. Ihler, R. L. Moses, M. J. Wainwright, and A. S. Willsky, "Distributed fusion in sensor networks," IEEE Signal Processing Magazine, vol. 23, no. 4, pp. 42-55, 2006.
[21] Y. Weng, R. Negi, and M. D. Ilić, "Graphical model for state estimation in electric power systems," in 2013 IEEE International Conference on Smart Grid Communications (SmartGridComm). IEEE, 2013, pp. 103108.

[22] S. L. Lauritzen, Graphical models. Clarendon Press, 1996, vol. 17.

[23] G. V. Moustakides et al., "Optimal stopping times for detecting changes in distributions," The Annals of Statistics, vol. 14, no. 4, pp. 1379-1387, 1986.

[24] J. Liu and J. Ye, "Moreau-yosida regularization for grouped tree structure learning," in Advances in neural information processing systems, 2010, pp. $1459-1467$.

[25] Y. Weng, Y. Liao, and R. Rajagopal, "Distributed energy resources topology identification via graphical modeling," IEEE Transactions on Power Systems, vol. 32, no. 4, pp. 2682-2694, 2016.

[26] V. Y. Tan, A. Anandkumar, and A. S. Willsky, "Learning gaussian tree models: Analysis of error exponents and extremal structures," IEEE Transactions on Signal Processing, vol. 58, no. 5, pp. 2701-2714, 2010. 